\title{
An Evidence-Based Medicine Approach to the Evaluation of the Role of Exogenous Risk Factors in Sporadic Amyotrophic Lateral Sclerosis
}

\author{
Carmel Armon \\ Department of Neurology, Loma Linda University School of Medicine, Loma Linda, Calif., USA
}

\section{Key Words}

Motor neuron disease $\cdot$ Evidence-based medicine $\cdot$ Risk factors · Amyotrophic lateral sclerosis $\cdot$ Case-control studies · Cohort studies · Mortality studies · Occupations accord priority to investigating putative risk factors with greatest public health impact. Advances in study methodology may lead to development of finite research cycles for individual putative risk factors for sporadic ALS.

Copyright $@ 2003$ S. Karger AG, Basel

\section{Introduction}

Amyotrophic lateral sclerosis (ALS) is the most common neurodegenerative disorder of motor neurons. Loss of pyramidal, spinal and bulbar motor neurons affecting multiple regions of the body leads to progressive motor dysfunction, disability and death. Five recent reviews of the epidemiology of ALS [1-5], as well as several book chapters [6-8], have considered environmental risk factors for ALS. They all came to similar conclusions: no association of an exogenous environmental risk factor with ALS has been demonstrated consistently and convincingly. However, the past 10 years have seen a significant shift in the focus of analytic studies looking for causes of sporadic ALS. First, there has been a shift away from looking for risk factors in the form of isolated events and towards looking for them in the form of chronic, lifelong, exposures. This shift has been driven chiefly by considerations of biologic plausibility and the need to assure that risk factors preceded disease onset. Second, the range of exposures under consideration has been expanded to include not only environmental factors but also lifestyle-

\begin{tabular}{ll}
\hline KARGER & ( ) 2003 S. Karger AG, Basel \\
0251-5350/03/0224-0217\$19.50/0 \\
$\begin{array}{l}\text { Fax +4161306 12 34 } \\
\begin{array}{l}\text { E-Mail karger@karger.ch } \\
\text { www.karger.com }\end{array}\end{array}$ & $\begin{array}{l}\text { Accessible online at: } \\
\text { www.karger.com/ned }\end{array}$
\end{tabular}

Carmel Armon, MD

Department of Neurology, Loma Linda University School of Medicine

11175 Campus Street, CP-11108

Loma Linda, CA 92354 (USA)

Tel.+1909 5584907, Fax +1 909 5580207, E-Mail res0tna7@verizon.net 
related exogenous factors, such as diet or smoking. Third, there is increased emphasis on the genetic-environmental interaction model, with time, or age, as a modifier [9].

In addition, there have been advances in the methodology of analyzing the published literature. Readers of epidemiological reviews and chapters 15 and 20 years ago may have encountered what today might be considered relatively uncritical summaries of the published literature. Between 15 and 10 years ago, the idea of a critical review of the literature began to impact the way data were summarized and presented: not all reports were accorded equal weight. Approximately 10 years ago, meta-analysis was considered for its utility to summarize and condense the results of many studies, which individually might be ambiguous or conflicting. However, limitations of this method when the component studies were less than perfect or comparable have not permitted its proper application to the epidemiology of ALS.

The past 10 years have seen the emergence of a group of core concepts known as 'evidence-based medicine' (EBM) and its incorporation into the way we think about drawing conclusions from the published literature. The application of these concepts has not been done in a uniform fashion. It varies among professional organizations and has undergone changes and refinements over time within individual organizations, including, for example, the American Academy of Neurology. There are advantages to applying this method to the analytic epidemiology of ALS. EBM makes explicit the expectation that review of data in the literature is done for the purpose of drawing conclusions and how that may be done. Where that is not possible, the purpose of the review is to determine what additional data would need to be generated in order to be able to draw conclusions in future ('directions for future research'). This would counteract the potential for 'circular epidemiology' [10]. EBM determines that all data are not equal as far as the ability to draw conclusions from them and shows why. EBM concerns itself with the extent to which data, derived from subsets or samples of the general population, may be used to draw inferences that may apply to the general population. This translates into evaluation for freedom from biases and generalizability. These concerns parallel the concepts of 'internal validity' and 'external validity' in traditional epidemiology and clinical trial research. EBM makes explicit how data are classified, and how firm a conclusion can be drawn from data that cross a threshold of conclusion-worthiness. As a result, if agreement is established with regard to the way the method is to be applied, the results of its application tend to be reproducible.
However, EBM has its limitations. There is more than one way to apply EBM, and the choice of the way it is applied is based on opinion, rather than on evidence. Conclusions may depend on which data are allowed to impact on them. Further, the choice of how EBM is to be applied is made with some knowledge of the data that is to be evaluated. This differs from the way data are derived in a research setting, where the experiment is done, and the results become known, only after the methods have been established. Moreover, EBM may favor data obtained from well-funded, well-designed clinical trials that demonstrate small effects convincingly over observations of effects of larger magnitude, if obtained and documented using less rigorous methodology. Stated otherwise, the availability of data to meet the requirements of EBM is subject to bias resulting from commercial interests. Finally, EBM is prone to type II errors (avoiding making a recommendation or drawing a conclusion when one is warranted) even as it tries to decrease the likelihood of type I errors (making a recommendation or drawing a conclusion with insufficient basis). In contrast to other research efforts and, in particular, clinical trials, it is usually impossible to estimate the magnitude of the likelihood of a type II error when applying EBM methods, whereas the likelihood of a type I error is usually explicit, and kept low. Thus, the impact of this particular limitation is hard to assess prospectively. A type II error in the application of EBM is only discovered in retrospect. Consequently, it is important to keep in mind that in EBM as in clinical trials, absence of evidence should not be construed automatically as evidence of absence. However, the advantages of utilizing the methods of EBM outweigh their potential limitations, particularly when these potential limitations are recognized.

In order to apply an EBM approach to evaluate the role of exogenous risk factors in sporadic ALS, it is necessary to have a rating system to classify the quality of evidence in published literature and to translate the evidence into conclusions. I have drawn freely on the approaches of rating systems for the classification of evidence developed over the past few years by professional organizations, in particular the American Academy of Neurology, as reflected for example in two recent parameters [11, 12]. However, the purpose of this review is to generate scientific discussion, rather than to obligate one or other group of individuals or organizations to a particular viewpoint. Consequently, while this review has undergone pre-publication peer review, there has been no attempt to build a consensus around, or seek organizational endorsement for this particular approach to the application of EBM. 
Table 1. Proposed system for classification of evidence of risk factors in ALS: rating of analytic epidemiological articles

Class I: One of the following.

1 Prospective or retrospective cohort study with parallel controls

a Exposure, and hence assignment to the 'exposed' cohort, established before knowledge of diagnostic status, or without knowledge of diagnostic status, or confirmable independently of the knowledge of diagnostic status. Consideration of and accounting for possible misclassification.

b Unexposed cohort is appropriate to the risk factor in question, is wellmatched to the exposed cohort on factors other than the exposure, and is otherwise representative of the general population.

c Diagnosis of ALS made applying uniform efforts and criteria to exposed and unexposed cohorts.

d Loss to follow-up low, and comparable in exposed and unexposed cohorts. Possible roles of competing causes of mortality accounted for. Preferably - all mortality data available for both cohorts.

e Exposure quantified, where possible, to permit assessment of doseresponse relationships.

f Sources of biases and confounding identified and accounted for.

$\mathrm{g}$ Conclusion based on large numbers. Appropriate statistical analysis.

2 Population-based case-control studies

a Putative risk factor or exposure occurred before probable biologic onset of disease

b Demonstration that ascertainment of patients is complete in the given population.

c Appropriate choice of controls, to assure they are matched to the patients and are also representative of the general population (assure adequate matching, avoid 'overmatching').

d High response rates from patients and controls.

e Uniform effort to gather information equally from affected and unaffected individuals.

f Blinding of information-gathering method to individuals' disease status ideal; if not done - adequate justification as to why this does not affect the assessment of the risk factor in question.

$\mathrm{g}$ Blinding of subjects and individuals gathering the data as to the hypotheses being tested. If not done - adequate justification as to why this does not affect the assessment of the risk factor in question.

$\mathrm{h}$ Meticulous attention to avoiding recall bias or, if not possible, to evaluating its impact, estimating the magnitude of its impact and controlling for it.

i Diagnosis of ALS made applying established criteria.

j Exposure quantified, where possible, to permit assessment of doseresponse relationships.

$\mathrm{k}$ Sources of biases and confounding identified and accounted for.

1 Conclusions based on large numbers. Appropriate statistical analysis. Methods state if hypotheses were selected a priori for confirmatory analysis. If more than one exposure considered in exploratory analysis, statistical significance is established with correction for multiple comparisons.

Class II

1 Cohort studies with parallel controls meeting most of criteria b-g, where the findings may be considered valid for the risk factor in question. This requires justification (criterion a is mandatory).

2 Population-based case-control studies meeting most of criteria c-l, where the findings may be considered valid for the risk factor in question. This requires justification (criteria $\mathrm{a}$ and $\mathrm{b}$ are mandatory).

3 Well-designed case-control studies that are not population-based, meeting most of criteria $\mathrm{c}-1$. Criterion a is mandatory. Justification is necessary, why the findings may be considered valid for the risk factor in question, with initial attention to referral bias.

\section{Class III}

1 Cohort studies with parallel controls where not all of the criteria b-g have been met, and consequently bias or confounding may account for the findings with regard to the risk factor in question, but not to an extent that would invalidate the findings completely.

2 Case-control studies where not all the criteria b-1 have been met, and consequently bias and confounding may account for the findings with regard to the risk factor in question, but not to an extent that would invalidate the findings completely.

Findings which result from otherwise unbiased exploratory analysis, or encountered through the performance of multiple comparisons, may belong to this class, provided no sources of bias or other material limitations are present. If there are additional limitations, then the evidence is class IV or V. Assignment to level III requires justification.

Class IV

All other studies with controls, where the risk factor occurred before biological disease onset. Results that do not attain statistical significance. Results of post-hoc analyses, uncorrected for implicit multiple comparisons.

\section{Class $V$}

1 Studies with controls where the risk factor studied most likely occurred after biological disease onset. Assignment to this class is specific to that risk factor.

2 Uncontrolled data (case series, case reports, chance observations, expert opinions that are not based on verifiable data).

Comment: Epidemiologic studies are designed to look for presence and magnitudes of associations. Findings of absence of association require special consideration. Absence of evidence (of association) is not equivalent to evidence of absence. In general, power calculations are needed to provide an estimate of the type II error (the likelihood of missing a true association where one is present). This information is needed in order to know how likely it is that absence of association is due to chance or to a sample size too small to detect a true effect of a predetermined magnitude. If power calculations are available, failure to find an association may be construed as class I or class II evidence in support of a conclusion that there is no association if the power is sufficiently large, typically $>80 \%$. In the absence of power calculations, failure to find an association might be considered at most as class III evidence in support of a conclusion that there is no association. However, power calculations are usually not performed when designing epidemiologic studies, and do not guide sample size. In fact, sample size is usually determined by practical constraints of resources (time and budget). Power calculations are not provided in published reports, and may be difficult to derive in retrospect.

Therefore, I am proposing an additional criterion that might permit considering failure to find an association as (at most) class II evidence for lack of association. It has two elements: (a) the finding is based on a large sample of patients and a large proportion of patients in the sample and (b) the $95 \% \mathrm{CI}$ around the OR of 1.0 is 'tight'. There are no universally accepted definitions of 'large number of patients' or of 'tight $95 \%$ CI'. I propose that the number of patients may be considered 'large' if the actual number of patients in the sample is $>50$ and the proportion of patients be considered 'large' if all, or close to all, the patients in the sample inform the conclusion. A $95 \% \mathrm{CI}$ round 1.0 may be considered 'tight' if its upper limit (UL) is $<2.0$ (to conclude 'no increased risk') or if its lower limit (LL) is $>0.5$ (to conclude 'no protective effect'). If this criterion is to be applied, then the number and proportion of patients on whom this conclusion is based should be provided, as well as the actual $95 \% \mathrm{CI}$, so that readers may decide how robust they consider the conclusion. 
Table 2. Translating evidence implicating an alleged risk factor for ALS into conclusions

Level A rating: This is an established risk factor ('overwhelming evidence')

Two class I studies, or one class I and two class II studies, or three class II

studies, with no contradictory evidence of equal or higher quality ranking, and which lend themselves to the application of the criteria for inferring causation from association [13]

Level B rating: This is a probable risk factor ('more likely than not')

1 One class I study or two class II studies, with no contradictory evidence of equal or higher quality ranking, and which lend themselves to the application of the criteria for inferring causation from association [13].

2 Two class I studies, or one class I and two class II studies, or three class II studies, with some contradictory evidence of equal quality ranking. The evidence in favor of inferring risk factor status preponderates, through the application of the criteria for inferring causation from association [13].

Level C rating: This is a possible risk factor (does not attain a 'more likely than not' status). Better-designed studies may be warranted with regard to this risk factor

One class II study or several class III studies with or without contradictory evidence of equal or lesser quality. If there is contradictory evidence of equal quality ranking, there must be less such evidence than there is evidence favoring risk factor status. The inconsistencies in the evidence do not permit consideration of inferences about causation.

Biologic plausibility is not necessary for assignment to this category.

Level U rating: It is unknown whether this is a risk factor

Evidence with regard to this risk factor is from conflicting or an insufficient number of class I-III studies, without a preponderance of evidence one way or another; or is from class IV-V studies; or there is no evidence.

If evidence that this is not a risk factor outweighs the evidence in support of this being a risk factor, assignment should be to a level A or B rating, as outlined in the comments below.

The specific criteria I am proposing for the classification of evidence are presented in table 1 . The criteria for drawing conclusions from the evidence are presented in table 2. For assignment of higher levels of certainty, table 2 incorporates the considerations put forward by Hill [13] and the US Surgeon General [14] for inferring causation from association, even though we are dealing with alleged risk factors, rather than alleged causes. In addition, a third set of criteria is presented, in anticipation of the possibility that some results may cross various thresholds of conclusion-worthiness, in order to decide how to rank their potential importance to the general public (table 3). Finally, I will state which developments in study methodology might result in higher quality studies in future, to make the research cycle with regard to specific risk factors finite. I expect the approach applied here to be critiqued, resulting in its future refinement. The limitations noted in many of the case-control studies reported before 1990 resulted in reflection of greater scientific rigor in some of the studies reported in the last decade. Hence, this review is limited to analytic studies reported from 1991 to 2002 that resulted in at least class III evidence.
Comments

1 This table is designed to permit moving from the default position, that it is not known if a putative risk factor is a risk factor (level U), to a position that a risk factor has been identified. Consistent evidence in the form of class I-III studies that permit inferring lack of association from failure to find an association (see comment for table 1) should also result in a shift away from level U. However, assignment to level C ('possibly not a risk factor') is meaningless in this setting. Hence, to translate evidence that there is no association, a simpler system is proposed, whereby a level B rating ("probably not a risk factor') is assigned if there is stronger quality of evidence that there is no association than that there is an association, and a level A rating ('definitely not a risk factor') is assigned if there is a preponderance of evidence for lack of association. Further, it should be recognized that, realistically, there will be very little impetus for the scientific and funding communities to replicate even one class II study that establishes that there is no association for a particular presumptive risk factor; hence, one class II study unopposed by equal or higher class evidence is a realistic minimal requirement for assignment of a level $\mathrm{B}$ rating for absence of association. This is a small relaxation of the requirements to establish level $\mathrm{B}$ rating in support of presence of association.

2 Biologic onset of ALS precedes clinical onset, but it is not known by how many years. In all likelihood, the slower the disease progression, the longer it is reasonable to assume its preclinical course has been. Exposures or events which happened within the 1-3 years before clinical onset of ALS most likely happened after its biological onset, thus cannot have caused the disease, and constitute class V evidence for risk factor status. Studies may choose to exclude from consideration exposures or events within 5 or even 10 years of clinical onset, to avoid this limitation.

3 Invoking Hill's criteria of inferring causation from association [13] to the process of assignment of risk factor level is done intentionally, recognizing that 'risk factor' and 'cause' are not synonymous. The purpose is that those risk factors that are assigned higher levels will be further along the road towards potential causal status than if they were mere associations.

4 Biologic plausibility. In general, a critical link between a well-established risk factor and causation is biologic plausibility. Biologic plausibility is proved either by producing the disease in excess in an appropriate animal model exposed to the risk factor or by knowing with certainty how the risk factor would interact with the established biological mechanism of disease causation. In the case of ALS, the biologic mechanisms underlying disease causation are not known, animal models of sporadic ALS are lacking, and the animal models of familial ALS may or may not be relevant to the pathogenesis of sporadic ALS. Thus, biological plausibility cannot be proved for most risk factors for ALS under consideration. However, biologic plausibility may be considered within the framework of existing hypotheses regarding the pathogenesis of ALS, recognizing the speculative or hypothetical nature of this process, for the time being. This is required for levels A and $\mathrm{B}$.

\section{Methods}

\section{Literature Identification}

The list of articles published in or after 1991, considered within the recently-published review by the Agency for Toxic Substances and Disease Registry (ATSDR) [5], served as a starting point. It was found to include relevant articles considered within previous reviews [1-4]. Additional articles, reporting results from well-designed studies so identified, were added to the list. An independent Medline literature search was performed to confirm the completeness of this method of article identification and to identify articles published in 2002, utilizing combinations of the key words ('amyotrophic lateral sclerosis' or 'motor neuron disease'), with 'risk factor' ('cohort' or 'case-control'), or 'environment.' 
Table 3. Considerations in ranking the importance of risk factors in ALS

1 Level of establishment of the risk factor status

a Greater weight given to a higher level ('established' and 'probable')

b Some weight given to 'possible,' with appropriate caveats

c No weight given to 'unknown'

2 What is the public health impact of the risk factor?

a Greater weight given if a large percent of the population is impacted

b Some weight given even if only a small percent of the population is affected

3 Is the risk factor modifiable?

a Greater weight given to modifiable risk factors

b Some weight given to non-modifiable risk factors

4 Does the risk factor have co-morbidities unrelated to ALS, or does it have 'redeeming features?'

a Greater weight given to risk factor with known co-morbidities unrelated to ALS or no 'redeeming features'

b Lesser weight given if risk factor for ALS may be protective for other, more prevalent conditions, which might cause earlier morbidity and mortality

\section{Classification of Evidence}

The full text of identified analytic articles published between 1991 and 2002 was reviewed, and the criteria in table 1 applied to classify the evidence in them. Articles providing at least class III evidence are discussed in detail and referenced here. Several articles resulting from one study are considered together. In general, articles classified as class IV or V evidence are not referenced here or considered further. The exception is when a particular study provided some evidence of class III or higher, and some evidence of class IV-V.

\section{From Evidence to Conclusions}

The criteria of table 2 were used to draw conclusions, and the considerations of table 3 to infer the broader impact of these conclusions.

\section{Opinions}

This article concludes with my opinions regarding (a) lessons learned from this review, and (b) directions for future research.

\section{Results}

\section{Literature Identification}

Twenty-eight articles published in or after 1991 were identified in a preliminary fashion from the ATSDR review [5]. Two were excluded because they did not have comparison groups. Eighteen additional articles were considered to provide only class IV-V evidence. Eight articles are considered further [15-22]. Four related articles were identified [23-26]. The Medline search identified one additional article published between 1991 and 2000, which did not add to material published in an article considered previously to provide class IV evidence, and four articles published in 2002, two of which provided class III evidence or better [26, 27]; one [26] had been identified previously.

\section{Classification of the Evidence}

Of the thirteen articles, eleven resulted from 5 casecontrol studies. One study was reported in five articles, one study in three articles, and three studies were each reported in one article. In addition, there were two population-based studies which analyzed correlations between disease-specific mortality and occupations. The case-control studies will be discussed first.

\section{Population-Based Case-Control Study in Western}

Washington State 1990-1994

This was a rigorously designed population-based casecontrol study, which followed on an incidence study [28], and resulted in five articles [17, 20, 21, 23, 24]. Such a study would be expected to provide class I evidence (see table 1). However, the published methods state that interviewers were not blinded to disease status of respondents, do not state whether interviewers were aware of any of the hypotheses being tested, do not state the total number of hypotheses tested, and do not state if any hypotheses were designated a priori as confirmatory (vs. exploratory). Furthermore, approximately one third of eligible controls refused to participate. This resulted in $64.4 \%$ of controls who had more than high school education, compared to $52.3 \%$ of cases. Even though education was used as a covariate in the analysis to adjust for this difference, it would increase the likelihood to find exposures associated with jobs requiring less education in patients. Thus, in general, the highest level of evidence should be a conservative class II, and may be warranted for positive findings (presence of association) where there are strong elements to support an association not due to chance or bias (rather than the minimal threshold for statistical significance $\mathrm{p}<$ 0.05 or a $95 \%$ confidence interval (CI) which excludes $1.0)$ such as a particularly low $p$ value and a robust doseresponse effect. Class III or IV assignment is warranted for all other positive associations because of lack of control for the multiple hypotheses tested and the risk of recall bias by patients, except if it is clear that the exposure was assessed after biological disease onset, where a class $\mathrm{V}$ assignment is appropriate. Findings of absence of association require separate consideration (see table 1 
comment). This discussion depends on absence of other sources of bias that should result in assignment to a lower class of evidence. Using this approach, the study showed:

(1) An association with exposure to agricultural chemicals in men only (class III evidence) [20]. The authors also state that this finding should be considered exploratory. No associations were found for exposures to metals and solvents when relying on assessment by a panel of industrial hygienists evaluating job histories, blinded to participant status (class III evidence, due to upper limit (UL) $95 \%$ CI usually $>2.0$, and small numbers of patients and controls), though such associations were found when relying on self-report (class IV evidence, due to risk of recall bias).

(2) Overall no difference in physical activity between patients and controls (class II evidence) [23]. This classification is assigned because the absence of statistically significant association is based on findings derived from a study that was designed to be confirmatory, as reflected by the meticulous methods, even though this is not stated explicitly. Data were quantified from all 174 patients in the sample. In addition to the authors' own analyses, direct inspection shows that in multiple analyses, the proportions of patients grouped based on levels of activity are similar in patients and controls.

(3) Slightly more patients reporting participating in organized high school sports (class III evidence), and some indication (in exploratory analysis) of increased risk with greater levels of leisure physical activity approximately 40 years before disease onset (class IV evidence) [23]. These are interdependent data.

(4) No associations with a family history of neurodegenerative diseases other than ALS (class III evidence: small numbers, concern for underascertainment); an association with a positive family history of ALS was found, as expected [17].

(5) No associations with other factors, including history of physical trauma (fractures (class II), electric shocks (class II for 'no injury' shocks, class III for shocks associated with unconsciousness of burns), or surgeries (class II)), residence in rural areas (class II), and (all class III) travel to Western Pacific foci, history of polio, polio immunization or tetanus immunization [17]. Class II level was assigned if UL $95 \%$ CI was $<1.5$ and more than 50 of the 174 patients informed the result.

(6) An association with lifetime cigarette smoking (class II, because of a significant dose-response effect and little chance of these data being influenced by bias) [24].

(7) No association with alcohol consumption (class II, UL $95 \%$ CI $\leq 1.4$ after adjustment for education and smoking, approximately 54\% of 161 patients and 59\% of 321 controls reported alcohol consumption) [24].

(8) Putative dietary risk factors (high glutamate or fat intake increase risk, dietary fiber intake decreases risk) [21]. This is class $\mathrm{V}$ evidence, because the dietary history was obtained for the year before clinical onset, when the disease was likely to have been biologically active; hence the dietary survey cannot be construed as risk factors assessment. The findings may even reflect dietary adjustments made in response to preclinical disease. The multiple comparisons further limit the ability to make any inferences from these findings.

\section{New England 1993-1996 Case-Control Study}

This was a well-designed study which compared recently diagnosed patients recruited at two referral clinics to population controls identified by random digit dialing. The findings were reported in three publications $[19,25$, 26]. Such a study might yield class I evidence with regard to risk factors unlikely to be influenced by referral, selfselection and recall biases. However, there are limitations similar to those of the Washington County Study. For example, only $71 \%$ of eligible cases and $76 \%$ of eligible controls participated in the study. Of the controls, $78 \%$ had a level of education above high school, compared to $67 \%$ of cases $(p=0.005)$; however, cases tended to have a higher income 5 years before the interview date than controls $(p=0.082)$. It cannot be stated whether these differences in the samples reflect truly differences between the general patient and control populations. However, odds ratio (OR) calculations were adjusted for education (as well as other factors). These limitations suggest that, at best, a lower assignment of class of evidence (class II) is warranted even in cases where these biases are unlikely to have influenced the findings. If the likelihood of the impact of biases is high, then class IV assignment is appropriate. Dietary exposures were assessed for 5 years prior to the interview, and patients were enrolled within 2 years of initial diagnosis, which is equivalent, on the average, to within 3 years of clinical onset. Thus, dietary exposures were assessed, on the average, 2 years before clinical onset; however, the disease was probably biologically active at the time, at least in some patients (class V evidence). Other risk factors were assessed for periods that extended earlier, but also included periods of active disease, and the authors were careful to point out when they may have been detecting consequences of disease rather than its causes. An exception to these general rules is the evaluation of lead exposure, where the authors' intent to perform 'confirmatory' analysis is clear: the authors state 
that this was the primary intent of the study and they invested special effort to collect biological samples from patients and controls [26]. The issues in the lead exposure component of the study are not whether multiple comparisons may have resulted in positive findings due to chance alone, but the extent to which biases may have impacted the findings, and how biological samples in patients who are already ill should be interpreted: Do they reflect the cause of the disease or its consequence? Applying these criteria, the New England Study showed:

(1) An association with cigarette smoking (class II evidence) [25]. A dose-response trend was demonstrated, but the level of statistical significance varied: it was 0.046 using 'cigarettes per day' as the measure, and 0.084 when using 'pack-years' as the measure. Statistical significance was not attained when using 'years smoked' as the measure; however, $12 \%$ of cases, compared to $4 \%$ of controls, had stopped smoking within the 5 years before the interview date. This likely reflects a case where the consequences of the disease interfered with continued exposure to the risk factor. The authors considered whether potential population controls who declined to participate may have impacted these findings, and reported that, compared to participants, refusers were less educated and less likely to have ever smoked. This increases the confidence in the findings that cases had greater frequency and quantity of smoking than controls.

(2) No association with alcohol use, marijuana use, or family history of neurologic disease other than ALS (class III evidence) [25]. An association with family history of ALS was found, as expected.

(3) Inconclusive data regarding dietary intake. Dietary intake of calcium and antioxidants were not strongly related to risk of ALS (authors' wording) (class V evidence) [20].

(4) An association of ALS with self-reported occupational exposure to lead and with elevated blood and bone lead levels, but no association with residential or recreational exposures. Adjusting for occupational exposures had little effect on the association of blood and patella levels, but weakened the association of tibia levels with ALS [26]. The latter observation suggests that blood and patella levels may not be good biological markers for lifetime lead exposure, or that exposure estimates based on the self-reported occupational histories are unreliable. The half-life for lead is 1 month in blood, 3-5 years in patella, and 15-25 years in tibia [26]. Tibia lead levels would thus be better markers for lifetime exposure, and adjusting for lifetime exposure would thus tend to decrease their association with ALS, as was found to be the case. The self-report results were driven by a small number of cases that provided the margin of excess occupational exposure: approximately 12 out of a total of 102 . Considering that 154 patients were eligible and 110 are listed as having participated, it is possible that this study of lead as a risk factor for ALS was enriched by patients with such an exposure, who may have been more willing to participate than those who did not have such an exposure. If the denominator had been 154 , rather than 102 , then the apparent excess would no longer be evident (35 out of $154=22.7 \%$, a proportion similar to 54 out of 247 controls $(21.8 \%)$ who reported exposure to lead fumes, dust or particles 10 or more times). Such a self-selection bias would also have enriched the proportion of patients with ALS with elevated blood and bone lead levels, if these were related to lifetime exposure. If the levels are a consequence of the disease rather than markers of its cause, this discussion is moot. Thus, the strength of evidence provided by this study for the association of exposure to lead with ALS is best assigned as class III. The authors conclude that 'the hypothesis that lead exposure plays a role in the etiology of ALS deserves further consideration', a conclusion that is consistent with class III evidence.

\section{Scottish Motor Neuron Disease Register 1990-1991}

Case-Control Study

This is a case-control study of 103 out of 147 patients with sporadic ALS diagnosed between May 1, 1990 and October 31, 1991, identified from the Scottish MND Register [16]. A structured interview was used to gather data. General practitioners' medical records for patients and controls were reviewed to supplement and corroborate the data from the interviews. The results may be summarized as follows:

(1) Results regarding an association of a history of fractures with ALS are inconclusive. A matched case-control analysis for lifetime history of fractures results in an OR of 1.3 , with a $95 \%$ CI of $0.7-2.5$. This does not attain statistical significance. The OR for men alone is 0.7 (a putative protective effect) and for women 2.8. Matched analysis for fractures within 5 years of symptom onset shows a highly significant excess in cases. However, in all likelihood, disease was biologically active at the time, and subclinical disease may have caused the fractures [1]. Subclinical ALS may predispose to fractures because of unanticipated muscle fatigue, or upper motor neuron dysfunction resulting in inducible spasticity, leading to an uncontrolled or poorly blocked fall which causes a fracture: the legs/arms/hands do not behave quite the way they are expected. Interestingly, if all 16 fractures within 5 years of 
clinical onset are excluded, there are 13 case-only couplets, compared to 20 control-only couplets, which might lead to a speculation that remote fractures reduce the likelihood of ALS. Thus, the entire association is based on class $\mathrm{V}$ evidence (risk factor likely occurred after biological disease onset). There was no correlation between site of fracture and the anatomical area in which the disease began.

(2) There was no significant difference between patients and controls in the number of non-bony traumatic events requiring medical attention, total number of operations or electric shocks (class IV evidence for lack of association, due to lack of actual numbers and CIs).

(3) An occupational history which included manual work, was more common in MND patients $(\mathrm{OR}=2.6$, 95\% CI 1.1-6.3). This was based on a detailed lifetime employment history classified according to the Office of Population and Censuses and Surveys (class III evidence, due to low likelihood of bias, but resulted from many comparisons).

(4) Regular exposure for a period over 12 months was reported by patients, more frequently than controls, for lead, chemicals/solvents, pesticides and minerals/ores. The 95\% CI excluded 1.0 for lead and chemicals/solvents (class IV evidence, due to risk of recall bias, difficulties quantifying the exposure, and risk of confounders).

(5) No statistically significant associations with environment in childhood, social class, common childhood infections, contact with poliomyelitis, vascular disease or tobacco use (class IV evidence for lack of association, due to limited data provided).

\section{Mayo Clinic, Rochester, Minnesota Referral-Based}

Case-Control Study

This case-control study was conducted in 1988-1989 utilizing a referral population at Mayo Clinic, Rochester, Minnesota [14]. It was designed to be a 'state-of-the-art' case-control study, and indeed tried to avoid many of the shortcomings of previous studies. Seventy-four patients were compared to 201 matched controls using a sequential questionnaire/interview technique to gather quantitative data regarding risk factors, with 5 hypotheses designated a priori as those for which the study would be considered confirmatory. Following the classification of evidence system (table 1), the results of such a study might be considered class II evidence. However, since I am evaluating my own work and due to methodologic limitations of the study, particularly when judged by today's standards, it may be more appropriate to represent the results as class III evidence. The study showed an association with greater expo- sure to lead and no associations of ALS with hard physical labor, trauma or major surgery, years lived in a rural community, or family history of neurodegenerative disease.

\section{Environmental Exposure to Trace Elements:}

\section{A Population-Based Case-Control Study}

This population-based case-control study was undertaken in the Emilia-Romagna region in Northern Italy [27]. Exposure to select trace elements was evaluated by measuring toenail concentrations in 22 patients and 44 controls. No association was found for the ten elements tested. There were no clearly identifiable sources of bias. However, toenail clippings reflect the time period of the preceding 12-15 months; hence, exposure was measured for a period during which ALS was biologically active, and possibly clinically evident. Consequently, the interpretation of the findings hinges on whether biological or clinical disease onset changed patients' exposure to or metabolism of the elements, in which case the toenail findings would not represent exposure before disease onset. Validation of toenails as biomarkers for chronic exposure has been provided for three of the elements (selenium, lead, zinc), but is less certain or unavailable for the others (cadmium, copper, aluminum, chromium, iron, cobalt, manganese). Nevertheless, the negative finding draws on the entire patient population a relative strength (class III evidence for lack of effect).

\section{Two Mortality Studies: Occupational Risk Factors}

The two studies under consideration were performed by correlating a diagnosis of ALS [18] or several discrete neurodegenerative diseases, including ALS [22], with occupational code in Swedish national [18] or United States multi-state [22] mortality databases. Different methods were used to determine relative excess frequency of diagnosis in different occupational groups. In the first study [18], an age-stratified random sample was selected as a control group, and for each occupation, the number of cases in that occupation was compared to an expected number, derived from the proportion of controls in that occupation. In the second study [22], the entire population served as the reference group: for each occupational group, the proportion of deaths from a specific cause was compared to the proportion of deaths from that cause in all occupational groups.

Both study designs are free of investigator bias. However, they are susceptible to two sources of bias at the stage of data entry into the surveillance systems. First is bias due to ascertainment and registration of the diagnosis, which may be more complete in some occupational 
groups than others. Ascertainment of diagnosis in the patient's life needs to carry over to awareness of the diagnosis by the physician registering it after the patient's death, and actually registering it. Ascertainment and registration will be influenced by level of sophistication, level of education, socioeconomic status, and overall level of initiative of patients and their surviving next of kin. Those who care are more likely to have the correct diagnosis made during life and registered after death. This consideration will matter less at the level of the individual in communities where there is high awareness and ascertainment of neurodegenerative diseases, and ALS in particular, but will undoubtedly play a role where awareness and ascertainment are less complete, specifically, in less advantaged socioeconomic settings. This awareness may also change over time. A second source of bias is the accuracy with which occupations are reflected on death certificates: not all occupations may be represented equally. This bias has been shown to happen with regards to electric utility workers [29]: even within a single industry, some occupations are more likely than others to be registered correctly on death certificates. Further, due to the multiple comparisons that take place as part of the study design, some associations will emerge as statistically significant due to chance alone, without reflecting on the operation of underlying etiologic processes. If ranking is performed, some occupations will rank higher than others, due to the study design. It is necessary to consider these potential sources of bias before considering the mortality/occupation study findings as truly representative of reality, let alone venturing to make etiologic inferences or speculations from such studies. Results based on small numbers of observed cases are particularly suspect because of their great proneness to bias, and may be assumed to be spurious or chance observations till proven otherwise. These study designs may generate at best class III evidence, if close attention is given to correcting for potential sources of bias; otherwise the evidence is class IV-V. In contrast, study designs that focus on single occupations can generate at best class IV evidence because the design itself makes them particularly prone to biases, including underascertainment in the reference population resulting in spuriously low expected numbers, with no way to control for these biases.

The 1991 mortality/occupation study from Sweden [18] compared the frequencies of occupations in patients with ALS who died between 1970 and 1983 to their frequencies in controls. Of 54 occupation codes reported, excess, defined as OR $>1.0$ and lower limit of $95 \%$ CI $>1.0$, was found for two occupations: office workers (34 cases, $\mathrm{OR}=1.8,95 \% \mathrm{CI} 1.0-3.3)$ and farm workers (56 cases, $\mathrm{OR}=1.7,95 \%$ CI 1.1-2.7). Fewer cases than expected were found among drivers ( 34 cases, $\mathrm{OR}=0.6$, 95\% CI 0.4-0.9) and chemical workers (12 cases, OR = $0.5,95 \%$ CI $0.2-1.0$ ). Additional occupations appeared to have greater or lesser than expected numbers of cases, based on small numbers. The category with the largest number of cases was farm and forestry managers (128 cases, $\mathrm{OR}=0.8,95 \% \mathrm{CI} 0.6-1.1)$. For women, the only excess was in medical services ( 33 cases, OR $=1.7,95 \%$ CI 1.0-3.0). The authors noted that the OR for agricultural work (farm managers, specialized and unskilled farm workers, 161 cases) versus all other branches of industry was 1.0, for each gender.

The study findings could be interpreted as having identified 'risky' occupations and 'safe' occupations, in which case the class of evidence for this interpretation should be class IV. Two out of 54 occupations in men and 1 out of 54 in women might be identified as risky, with a lower limit of $95 \%$ CI just $>1.0$, due to chance alone. This is without even getting into the question of the possible biases in reporting, discussed in the preceding paragraphs. An alternative interpretation of the findings is that overall no occupation stood out as excessively prone to dying of ALS, beyond what might be expected due to chance alone. In my opinion, the latter interpretation is more correct, and merits classification as class III evidence for lack of association of ALS with any particular occupation.

The 1996 study from the USA [22] examined mortality from 1982 to 1991 for 27 states, in four neurodegenerative disease (NDD) categories: presenile dementia, Alzheimer's disease, Parkinson's disease and motor neuron disease. The ten occupations with the highest proportional mortality ratios (PMRs), for each disease category, were listed for each of four demographic groups, designated by the authors as: white males, black males, white females and black females. Most occupations got into the 'top 10' based on small numbers of cases, usually $<10$. Teachers were represented with excess cases most consistently across all four disease categories, with large numbers of cases noted in 2 or 4 demographic groups for each disease category, PMRs ranging from 1.6 to 2.7, and lower limits of the $95 \%$ CI consistently $>1.0$. Other occupations represented as showing excess did so based on small numbers, and the interpretation of the findings is uncertain, due to potential for bias. Focusing specifically on motor neuron disease, the number of cases in the 'top 10' excess mortality occupations was $>10$ for two occupations for white men, eight occupations for white women, and one occupation each for black men and women. Of 462 white women in the 'top 10' excess mortality occupations, $227(49 \%)$ 
were teachers $(\mathrm{PMR}=1.6,95 \%$ CI 1.4-11.8). The PMR for black, female teachers was 2.1 (95\% CI 1.14-3.5, based on 14 cases). Male teachers did not make it into the 'top 10' occupations at risk for motor neuron disease; thus, the PMR for male teachers in this dataset is unavailable from the published report.

The study findings could be interpreted by focusing individually on some of the non-teaching occupations. Such focus would run the risk of being influenced heavily by the various biases previously discussed. Alternatively, the focus could be on the consistent excess representation of teachers across all categories of NDD - a more robust and consistent observation, affecting many more individuals. This focus could give rise to two possible lines of interpretation: (1) teachers who develop NDD are more likely than the average person to have both the diagnosis and their profession registered correctly on their death certificates, or (2) teachers are at greater than average risk for developing all NDD. Even if one was inclined to accept interpretation 2, before pursuing etiologic speculations related to the teaching occupation, it would be prudent to consider the possible role of reduced competition from other causes of mortality (Gompertzian considerations) [30] in producing the findings. The most conservative interpretation of the teachers' excess mortality data would be in terms of an apparent excess arising from more complete ascertainment of NDD and registration of disease and occupation in death certificates of teachers than of the reference population. Considering motor neuron disease specifically, since 1983-1991 US mortality (death certificate) data fell short of the Rochester, Minnesota incidence data by approximately $30-40 \%$, likely due to less than complete ascertainment, a PMR of 1.6 would bring the mortality in teachers to the expected range.

To summarize, the findings of this study could be interpreted in terms of the non-teaching occupations found to be at risk, based on small numbers, in which case the classification of this evidence would be class IV at best (due to the biases inherent in such an interpretation). Alternatively, the study could be interpreted as showing no excess risk in any particular occupation, because all apparent excesses might be explained by the operation of chance or biases. In my opinion, the latter interpretation is sounder, and would qualify as class III evidence for lack of association of occupation with ALS. A useful product of this approach is that we can use the 1.6-2.7 PMR in teachers as an approximate correction factor when trying to interpret apparent excesses that we believe may be due to more complete ascertainment in a special population under consideration than in the reference population.

\section{From Evidence to Conclusions}

Smoking has a probable positive association with ALS (level B rating, based on two class II studies [24, 25]). This risk factor warrants a high ranking of importance according to all four criteria in table 3 . It is considered 'probable,' it is modifiable, it has no redeeming features, and it affects a relatively large segment of the population. It is intriguing to speculate that some of the reduction of the male:female preponderance of ALS in more recent epidemiologic studies may be due to the rise in prevalence of smoking among women in the latter part of the last century. Smoking has not been thought of within the framework of 'environmental' risk factors in the past. Consequently, the significance of the two recent studies implicating it may not have had the same impact on the scientific and patient communities as even one study of equal quality implicating an exogenous, truly environmental, risk factor. There is some biologic plausibility to consider a role for smoking, a known carcinogen, in producing a condition that acts like a biochemical malignancy (fig. 1) [31]. Reduction in the ability to detoxify smoking-related carcinogens by some post-menopausal women [32] may explain the increased mortality from ALS predominantly in women over 65 in a recent report [33]. In additional support of a carcinogen, or alkylating agent, as possibly involved in causing some forms of ALS, Spencer [34, 35] currently favors methylazoxymethanol, a potent alkylating agent, the aglycone of cycasin, as the primary mechanism for the long-latency induction of Western Pacific ALS. He postulates that methylazoxymethanol may produce post-mitotic DNA damage and interfere with DNA repair, up-regulate the expression of $\tau$ mRNA by itself or in conjunction with an endogenous excitotoxic agent (neurotransmitter glutamate), and thus promote the accumulation of $\tau$ protein and neuronal degeneration in Western Pacific ALS/PDC.

The following are probably not risk factors for ALS (level B rating; supporting studies providing class II evidence listed; no class I evidence available): (1) trauma [17]; (2) physical activity [23]; (3) residence in rural areas [17], and (4) alcohol consumption [24].

Currently, there are no conflicting data of equal or higher evidence class. This rating may be revised if conflicting, high-quality data become available in future.

(1) Conclusions with regard to all other putative risk factors would attain at most a level $\mathrm{C}$ rating. Of these, exposure to lead and to agricultural chemicals have no redeeming features and are modifiable (i.e., preventable) but might end up affecting only a minority of patients. 
Fig. 1. Malignant biochemical transformation hypothesis. Risk factors operate 'upstream' to a malignant biochemical transformation which causes the appearance of 'endogenous ALS-specific toxins'. These putative toxins spread, behaving like a metastasizing biochemical malignancy, and cause the 'downstream' biochemical, histologic and clinical consequences of ALS [adapted from 31].

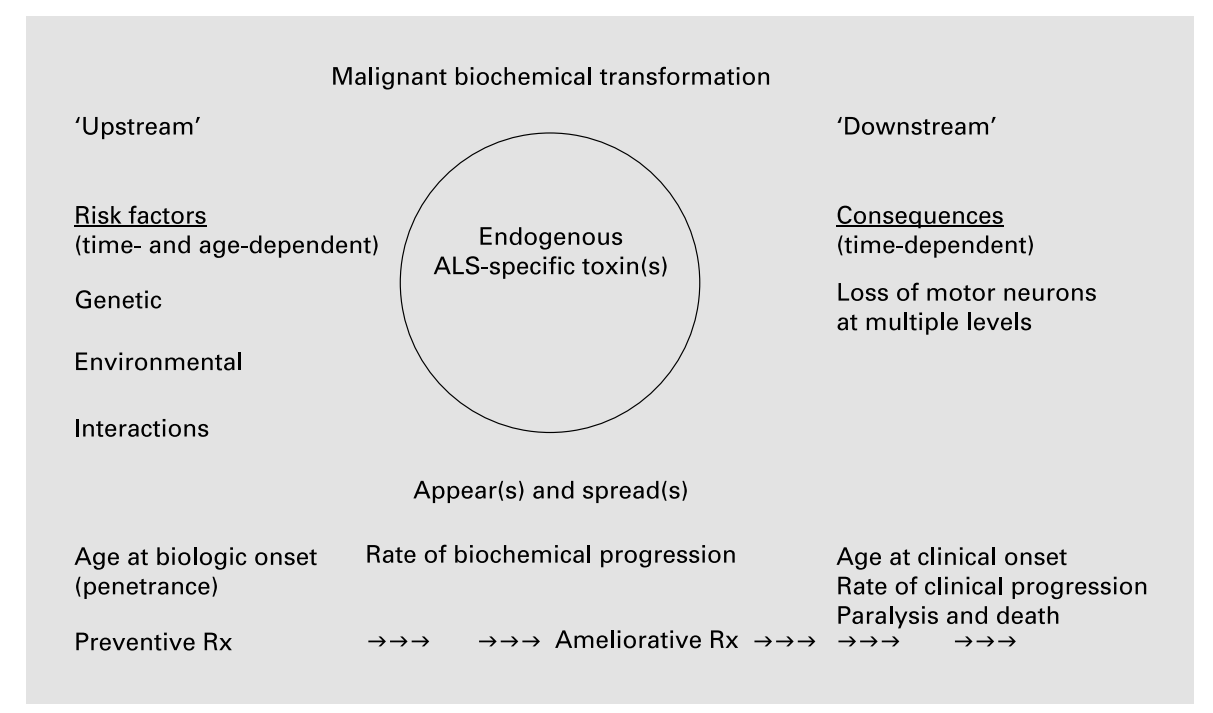

Dietary risk factors have aroused interest; however, the actual data (outside of Guam) are class V evidence and may affect only a small minority of patients. While it is intriguing to speculate that high glutamate intake (mainly due to high protein intake) may expose motor neurons to a chronic excitotoxic stimulus, this hypothesis could be tested directly by checking if different amounts of dietary glutamate change CSF glutamate in a small number of healthy volunteers. This has not been shown to occur in healthy dogs [36].

(2) It is impossible to take into consideration the likely underreporting or de-emphasizing of studies that have shown lack of associations.

\section{Opinions}

\section{Lessons Learned}

(1) Even the best, population-based, case-control studies are susceptible to biases.

(2) It may be very difficult, if not impossible, to design case-control studies to provide class II evidence in support of risk factors affecting only a small minority of patients, because the likelihood of bias is high, and the patient numbers are too small to generate reliable doseresponse data.

(3) It may be necessary to begin considering how cohort studies may be used in researching risk factors for ALS. It may be necessary to accept that the time unit for advances in this field is decades, or even investigator lifetimes, rather than 1- to 5-year grant-funding cycles.
(4) Mortality studies are subject to ascertainment and registration biases that can only be appreciated if internal and external controls are utilized.

(5) Findings based on small numbers of affected patients are likely to be incorrect.

(6) Findings based on large numbers of affected patients are likely to provide direction with regard to disease etiology or research methodology.

(7) It is necessary to reflect awareness that biologic disease onset precedes clinical onset by several years in design of studies of exogenous risk factors in ALS.

\section{Directions for Future Research}

(1) Development and publication of research methodology to determine how case-control studies can be designed with confirmatory intent to generate class I or class II evidence for or against associations of specific risk factors and ALS, in a way that would make them more similar to clinical trials. Consider the effect of having a priori clearly defined and formulated hypotheses and sample size calculations that will permit testing those hypotheses. Take into consideration: magnitude of increased risk of developing ALS that one wishes to be able to detect; magnitude of anticipated exposures; the percent population affected; anticipated responder rates and their impact; acceptable size of type I and type II errors.

(2) Development and publication of research methodology to determine what may be expected from cohort studies in ALS, following the same considerations as in point 1 . This may permit realistic planning of the scope and duration of cohort studies that might be undertaken 
with specific hypotheses in mind and with confirmatory intent.

(3) Establishment of priorities for putative risk factors to be studied (consider table 3).
(4) Development of research plans for the highest priority risk factors with study designs and time lines that will make the research of those factors a finite proposition [10].

\section{References}

1 Nelson LM: Epidemiology of ALS. Clin Neurosci 1995/96;3:327-331.

2 Roman GC: Neuroepidemiology of amyotrophic lateral sclerosis: Clues to aetiology and pathogenesis. J Neurol Neurosurg Psychiatry 1996;61:131-137.

3 Mitchell JD: Amyotrophic lateral sclerosis: Toxins and environment. Amyotroph Lateral Scler Other Motor Neuron Disord 2000;1:235250.

4 Armon C: Environmental risk factors for amyotrophic lateral sclerosis. Neuroepidemiology 2001;20:2-6.

5 Noonan CW, Sykes L, Hilsdon R: Motor neuron disease/amyotrophic lateral sclerosis: Preliminary Review of Environmental Risk Factors and Mortality in Bexar County, Texas. Agency for Toxic Substances and Disease Registry (March 5, 2002) http://www.atsdr. cdc.gov/NEWS/als_032002.html

6 Armon C, Kurland LT: Classic and Western Pacific Amyotrophic Lateral Sclerosis: Epidemiologic comparisons; in Hudson AJ (ed): Amyotrophic Lateral Sclerosis: Concepts in Etiology and Pathogenesis. Toronto, University of Toronto Press, 1990, pp 144-165.

7 Armon C, Kurland LT, Smith GE, Steele JS: Sporadic and Western Pacific Amyotrophic Lateral Sclerosis: Epidemiologic implications; in Smith RA (ed): Handbook of Amyotrophic Lateral Sclerosis. New York, Dekker, 1992, pp 93-131.

8 Armon C: Motor neuron disease; in Gorelick PB, Alter M (eds): Handbook of Neuroepidemiology. New York, Dekker, 1994, pp 407-456.

9 ALSA-Funded Workshop: Meeting Report. Environmental factors and genetic susceptibility in amyotrophic lateral sclerosis. May 29-31, 2002, Keystone, Colo. http://www.alsa.org/research/workshops1.cfm

10 Kuller LH: Circular epidemiology. Am J Epidemiol 1999;150:897-903.

11 Hirtz D, Ashwal S, Berg A, Bettis D, Camfield C, Camfield P, Crumrine P, Elterman R, Schneider S, Shinnar S: Practice parameter: Evaluating a first nonfebrile seizure in children. Report of the Quality Standards Subcommittee of the American Academy of Neurology, The Child Neurology Society, and The American Epilepsy Society. Neurology 2000;55:616623.

12 Lewis DW, Ashwal S, Dahl G, Dorbad D, Hirtz D, Prensky A, Jarjour I: Practice parameter: Evaluation of children and adolescents with recurrent headaches. Report of the Quality Standards Subcommittee of the American Academy of Neurology and the Practice Committee of the Child Neurology Society. Neurology 2002;59:490-498.
13 Hill AB: The environment and disease: Association or causation? Proc R Soc Med 1965;58: 295-300.

14 US Department of Health, Education and Welfare: Smoking and health: A report of the Surgeon General. Washington, US Government Printing Office, 1964.

15 Armon C, Kurland LT, Daube JR, O'Brien PC: Epidemiologic correlates of sporadic amyotrophic lateral sclerosis. Neurology 1991;41: 1077-1084.

16 Chancellor AM, Slattery JM, Fraser H, Warlow CP: Risk factors for motor neuron disease: A case-control study based on patients from the Scottish Motor Neuron Disease Register. J Neurol Neurosurg Psychiatry 1993;56:12001206.

17 Cruz DC, Nelson LM, McGuire V, Longstreth WT Jr: Physical trauma and family history of neurodegenerative diseases in amyotrophic lateral sclerosis: A population-based case-control study. Neuroepidemiology 1999;18:101-110.

18 Gunnarsson LG, Lindberg G, Soderfeldt B, Axelson O: Amyotrophic lateral sclerosis in Sweden in relation to occupation. Acta Neurol Scand 1991;83:394-398.

19 Longnecker MP, Kamel F, Umbach DM, Munsat TL, Shefner JM, Lansdell LW, Sandler DP: Dietary intake of calcium, magnesium and antioxidants in relation to risk of amyotrophic lateral sclerosis. Neuroepidemiology 2000;19: 210-216.

20 McGuire V, Longstreth WT Jr, Nelson LM, Koepsell TD, Checkoway H, Morgan MS, van Belle G: Occupational exposures and amyotrophic lateral sclerosis. A population-based case-control study. Am J Epidemiol 1997;145: 1076-1088.

21 Nelson LM, Matkin C, Longstreth WT Jr, McGuire V: Population-based case-control study of amyotrophic lateral sclerosis in western Washington State. II. Diet. Am J Epidemiol 2000;151:164-173.

22 Schulte PA, Burnett CA, Boeniger MF, Johnson J: Neurodegenerative diseases: Occupational occurrence and potential risk factors, 1982 through 1991. Am J Public Health 1996; $86: 1281-1288$

23 Longstreth WT, McGuire V, Koepsell TD, Wang Y, van Belle G: Risk of amyotrophic lateral sclerosis and history of physical activity: A population-based case-control study. Arch Neurol 1998:55:201-206.

24 Nelson LM, McGuire V, Longstreth WT Jr, Matkin C: Population-based case-control study of amyotrophic lateral sclerosis in western Washington State. I. Cigarette smoking and alcohol consumption. Am J Epidemiol 2000; 151:156-163.
25 Kamel F, Umbach DM, Munsat TL, Shefner JM, Sandler DP: Association of cigarette smoking with amyotrophic lateral sclerosis. Neuroepidemiology 1999;18:194-202.

26 Kamel 2002 Kamel F, Umbach DM, Munsat TL, Shefner JM, Hu H, Sandler DP: Lead exposure and amyotrophic lateral sclerosis. Epidemiology 2002;13:311-319.

27 Bergomi M, Vinceti M, Nacci G, Pietrini V, Bratter P, Alber D, Ferrari A, Vescovi L, Guidetti D, Sola P, Malagu S, Aramini C, Vivoli G: Environmental exposure to trace elements and risk of amyotrophic lateral sclerosis: A population-based case-control study. Environ Res 2002;89:116-123.

28 McGuire V, Longstreth WT Jr, Koepsell TD, van Belle G: Incidence of amyotrophic lateral sclerosis in three counties in western Washington State. Neurology 1996;47:571-573.

29 Andrews KW, Savitz DA: Accuracy of industry and occupation on death certificates of electric utility workers: Implications for epidemiologic studies of magnetic fields and cancer. Bioelectromagnetics 1999;20:512-518.

30 Riggs JE, Schochet S Jr: Rising mortality due to Parkinson's disease and amyotrophic lateral sclerosis: A manifestation of the competitive nature of human mortality. J Clin Epidemiol 1992;45:1007-1012.

31 Armon C: ALS: Clinical and epidemiologic clues to pathogenesis; in Neurobiology of ALS. Course Syllabus, 51st Annual Meeting, American Academy of Neurology, 1999.

32 Ambrosone CB, Freudenheim JL, Graham S, Marshall JR, Vena JE, Brasure JR, Michalek AM, Laughlin R, Nemoto T, Gillenwater KA, Shields PG: Cigarette smoking, N-acetyltransferase-2 genetic polymorphisms, and breast cancer risk. JAMA 1996;276:1494-1501.

33 Seljeseth YM, Vollset SE, Tysnes OB: Increasing mortality from amyotrophic lateral sclerosis in Norway? Neurology 2000;55:12621266.

34 Esclaire F, Kisby G, Spencer P, Milne J, Lesort $\mathrm{M}$, Hugon J: The Guam cycad toxin methylazoxymethanol damages neuronal DNA and modulates $\tau$ mRNA expression and excitotoxicity. Exp Neurol 1999;155:11-21.

35 Kisby GE, Kabel H, Hugon J, Spencer P: Damage and repair of nerve cell DNA in toxic stress. Drug Metab Rev 1999;31:589-618.

36 Schaeffer MC, Rogers QR, Leung PM, Wolfe BM, Strombeck DR: Changes in cerebrospinal fluid and plasma amino acid concentrations with elevated dietary protein concentration in dogs with portocaval shunts. Life Sci 1991;48: 2215-2223. 